Conclusion TASER appears to be a safe and efficient approach providing an optimal platform for resection of large rectal lesions. In our experience it provides the optimal platform for the minimally-invasive management of these high risk lesions. Disclosure of Interest None Declared.

\section{OC-049 RECTAL NEUROENDOCRINE TUMOURS: MANAGEMENT AND SURVIVAL IN 60 PATIENTS}

${ }^{1} \mathrm{~K}$ Naik*, ${ }^{2} \mathrm{R}$ Rossi, ${ }^{1} \mathrm{M}$ Caplin, ${ }^{1} \mathrm{C}$ Toumpanakis. ${ }^{1}$ Gastroenterology, Royal Free Hospital, London, UK; ' Gastroenterology, Postgraduate School of Gastroenterology, Universita' Degli Studi Di Milano, Milan, Italy

\subsection{6/gutjnl-2014-307263.49}

Introduction Rectal neuroendocrine tumours (rNETs) are increasing in incidence, with more found incidentally on routine colonoscopy. Our aim is to retrospectively analyse a cohort of rNETs to characterise diagnostic features and clinical behaviour.

Methods Patients (pts) with confirmed diagnosis of rNET were identified from a database.

Results 60 pts evaluated, median age 55 years (range 23-78). Most common presentation was rectal bleeding $\mathrm{n}=29$ (48\%). 29 / 60 pts had tumour $<1 \mathrm{~cm}, 7 / 60$ pts $1-2 \mathrm{~cm}, 22 / 60>2 \mathrm{~cm}, 2 / 60$ size was unknown. Of patients with tumour size $<1 \mathrm{~cm}, 3 / 29 \mathrm{did}$ not require endoscopic follow-up (pT1a) and of the other 26, none had evidence of recurrence on endoscopic follow-up (follow-up range 6 to $88 \mathrm{~m}$ ). 24/60 pts had metastases at presentation, $5 / 60$ developed metastases during follow-up (of these 29 pts $86 \%$ liver, $40 \%$ bone, $10 \%$ lung). Of 29 pts with metastases, $24 / 29$ had somatostatin receptor imaging with $62 \%$ avid uptake. Chromogranin A available in $23 / 29$ pts: not elevated in $83 \%$. Of 29 pts with metastases, 19/29 had chemotherapy, 10/29 somatostatin analogues (SST), 15/29 surgery and 10/29 peptide-receptor-radionuclide-therapy (PRRT). Chemotherapy: 1/19 pts partial response, 2/ 19 stable disease (SD), 12/19 progressive disease (PD) (median time to progression 4 months $(\mathrm{m})$ ); 4/19 no data. PRRT: 4/10 had $\mathrm{SD}$ (follow-up range 24 to $53 \mathrm{~m}$ ), 4/10 PD (median time to progression $4 \mathrm{~m}$, range 2-9), 2/10 no data. SST: 2 sustained SD (range 12-27 m), 7/10 PD, (median time to progression 3m, range 2-5); $1 / 10$ no data. During median follow-up of $20 \mathrm{~m}$ (range 3-170 m), $100 \%$ of pts with primary tumour $<1 \mathrm{~cm}, 86 \%$ with tumour size $1-2 \mathrm{~cm}$, and $25 \%$ with size $>2 \mathrm{~cm}$ are currently alive. Tumour size $>2 \mathrm{~cm}$ have poorer outcome than the other 2 groups $(\mathrm{p}<0.001)$. Conclusion Tumours $>2 \mathrm{~cm}$ are associated with poor prognosis. Chromogranin A is mostly normal even in advanced disease. Prospective studies are needed to determine progression free survival data for systemic therapy.

Disclosure of Interest None Declared.

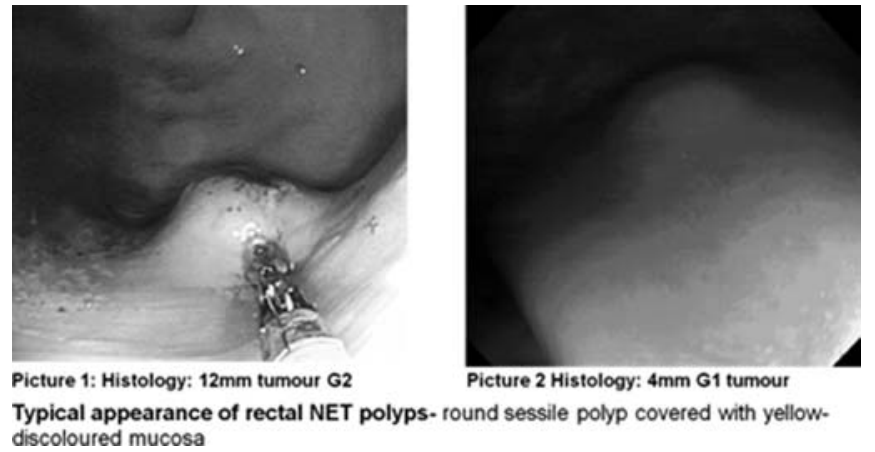

Abstract OC-049 Figure 1

\section{Trainee section symposium and free papers}

\section{OC-050 THE UGIB-DOPS: IMPROVING TRAINING IN GI BLEED MANAGEMENT IN THE ENDOSCOPY UNIT}

L China*, G Johnson. Gastroenterology, UCL, UCLH, London, UK

\subsection{6/gutjnl-2014-307263.50}

Introduction The 2007 GI Bleed Audit highlighted significant deficiencies and inconsistencies in service provision and care of patients presenting with UGIB. There is a pressure on UK hospitals to provide a $24 / 7$ endoscopy service to meet NICE guidance on timely endoscopic intervention in upper GI bleeding (UGIB), resulting in an urgent need to determine an endoscopist's competence. JAG provide quality assurance in UK endoscopy by using compulsory summative assessment in diagnostic endoscopy and more recently polypectomy.

There is currently no structured, formal tool or criteria with which to assess and provide feedback for the specific generic and endoscopic skills required for effective management of UGIB. DOPS are used as a tool to assess endoscopic skills by providing a framework for experts to observe, assess and provide feedback on a procedure. We developed a new DOPS tailored to the specific aspects of therapeutic endoscopic management of UGIB to improve training, with a view to developing the tool for use in summative assessment for JAG accreditation.

Methods A working group of expert endoscopists was formed at University College London Hospital. UGIB task deconstruction was undertaken and, after multiple revisions, consensus was reached on the individual aspects of management, and then to define what was considered a satisfactory endoscopic performance in each of these domains. The performance rating scale was based on the degree of independence demonstrated by the trainee in each performance domain. These aspects of performance, definitions of standards and rating scales were then used to construct the UGIB-DOPS.

We evaluated the feasibility, validity and educational impact of UGIB-DOPS using 8 trainees paired with trainers using questionnaires and semi-structured interviews.

Results The trainee cohort displayed a range of experience from novices $(n=2)$ to trainees who had managed $>80$ cases $(\mathrm{n}=2)$. Qualitative assessment of the educational impact of UGIB-DOPS found universal agreement that the tool's defined assessment criteria facilitated structured feedback and it was perceived the overall grade awarded reflected trainee's current competence. Thematic interview analysis revealed recurring concepts of how UGIB-DOPS facilitated training: creation of an observed teaching event, knowledge of the required

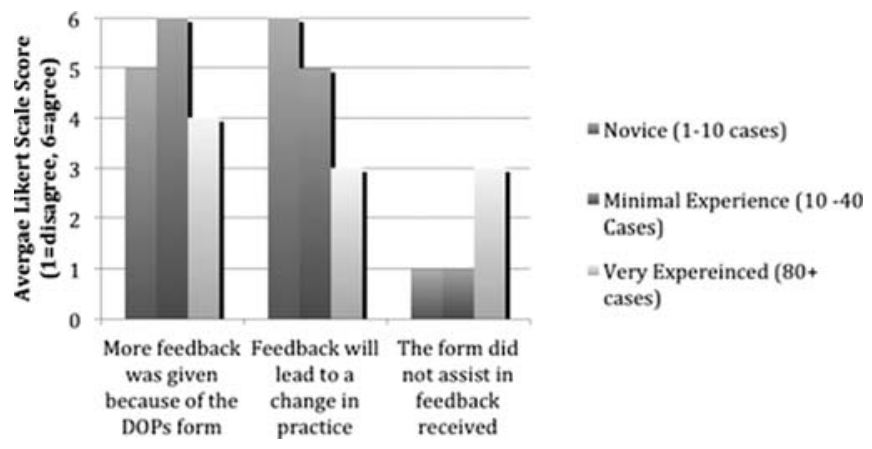

Abstract OC-050 Figure 1 